\title{
Plasma hyaluronic acid is increased in peripheral arterial disease and altered by fish oil supplementation
}

\author{
J. Madden ${ }^{1}$, C. Shearman ${ }^{2}$, R. F. Grimble ${ }^{2}$ and P. C. Calder ${ }^{2}$ \\ ${ }^{1}$ Department of food and Nutritional Sciences, University of Reading, RG6 6AP, UK and ${ }^{2}$ Developmental origins of \\ health and disease, University of Southampton, SO16 6YD, UK
}

Hyaluroinic acid (HA) is an intra-cellular matrix polymer whose main functions are structural and the maintenance of tissue hydration. The catabolism of the HA polymer to smaller subunits is initiated in membrane lipid rafts and is dependent on CD44 ${ }^{(1)}$. These smaller subunits are released into circulation and are thought to be proinflammatory as plasma levels increase in acute inflammatory conditions such as septicaemia ${ }^{(1)}$. Plasma HA levels are also a strong indicator of severe liver fibrosis as fibrotic cytokines such as TGF and FGF enhance HA catabolism ${ }^{(2)}$.

We hypothesised that circulating HA levels would be increased in peripheral arterial disease (PAD) as this disease it is known to have both fibrotic and inflammatory components. Furthermore, as fish oil supplementation has been shown to reduce inflammation and monocyte CD44 expression ${ }^{(3)}$, we investigated the effect of supplementation of $1.7 \mathrm{~g}$ EPA + DHA per day for 12 weeks on plasma HA levels in 72 PAD patients.

In 83 healthy controls, we found plasma HA levels to be low (mean $1.54 \mathrm{ng} / \mathrm{ml} \pm 1.35$ ), whereas in 72 PAD patients plasma HA levels were much higher (mean $19.75 \mathrm{ng} / \mathrm{ml} \pm 3.76)(P<0.001)$. Following fish oil supplementation in the PAD group plasma HA levels increased (mean $27.03 \mathrm{ng} / \mathrm{ml} \pm 5.64)(P=0.02)$.

One possible mechanism for these findings is that fish oil has previously been shown to increase expression of a splice variant form of CD44, CD44v3, on PAD monocytes ${ }^{(3)}$. CD44v3 can bind fibrotic cytokines such as TGF and FGF, and may increase their potency leading to increased fibrosis and stabilisation of atherosclerotic plaques ${ }^{(4)}$.

Another explanation is that increased circulating HA levels are often seen in high-volume blood loss and thought to act as a intravascular volume expander, so preventing circulatory collapse. In vascular compromised individuals, increased levels of HA may enhance circulatory competence which we have previously demonstrated for fish oil supplementation in PAD ${ }^{(5)}$.

1. Noble PW (2002) Matrix Biol 21, $25 \mathrm{e} 9$.

2. Avila RE, Carmo RA, Farah Kde P et al. (2010) JR Braz J Infect Dis 14, 335-341.

3. Madden J, Shearman CP, Dunn RL et al. (2009) Nutr Metab Cardiovasc Dis 19, 247-252.

4. Thies F, Garry JM, Yaqoob P et al. (2003) Lancet 361, 477-485.

5. Madden J, Brunner A, Dastur ND et al. (2007) Prostaglandins, Leukotriens and essential fatty acids 76, 331-340. 